\title{
Wettbewerbselemente zur Stärkung der Versorgungsqualität
}

CHRISTOPHER HERMANN, JÜRGEN GRAF

Dr. Christopher Hermann ist Vorstandsvorsitzender der AOK Baden-Württemberg in Stuttgart

Jürgen Graf ist Fachbereichsleiter „Integriertes Leistungsmanagement" bei der AOK Baden-Württemberg in Stuttgart

\begin{abstract}
Das vor 20 Jahren verabschiedete GesundheitsStrukturgesetz sticht als Gesetzgebungsverfahren hervor, das nachhaltige Strukturveränderungen durch Versicherungswahlfreiheit bewirkt hat. Im Bereich der Leistungserbringung und Versorgungsgestaltung erfolgten keine auch nur annähernd tiefgreifenden Veränderungen. Im Ergebnis können die jeweils punktuellen und am System korporatistischer Selbstverwaltung orientierten Bemühungen den Erfordernissen einer Bevölkerung mit steigenden Prävalenzen chronischer Erkrankungen in einer Gesellschaft des längeren Lebens nicht gerecht werden. Es ist hohe Zeit, die Krankenkassen auch in einen Versorgungswettbewerb zu stellen, innen Instrumente zur Verfügung zu stellen und dem Kassenwettbewerb endlich patienten- und versorgungsorientierten „Drive“ zu geben.
\end{abstract}

\section{Reformnotwendigkeit}

Das aktuelle Positionspapier „Wettbewerb, Sicherstellung, Honorierung Neuordnung der Versorgung im deutschen Gesundheitswesen“ der FriedrichEbert-Stiftung bringt es nochmals auf den Punkt: „Die Zunahme chronischer und psychischer Erkrankungen, die demografische Entwicklung und der medizinische Fortschritt machen die Förderung integrierter Versorgungsformen erforderlich. Zugleich müssen Primärversorgung gestärkt und die hausarztzentrierte Versorgung ausgebaut werden“, und weiter: „Das System korporatistischer Selbstverwaltung war bisher nicht in der Lage, die aktuellen Versorgungsdisparitäten zu lösen“.

Bereits mit dem wegweisenden Gutachten 2000/2001 des Sachverständigenrats zur Begutachtung der Entwicklung im Gesundheitswesen (SVR) unter dem Motto „Bedarfsgerechtigkeit und Wirtschaftlichkeit" (Über-Unter-
Fehlversorgung) sowie der nachfolgenden Gutachten mit den Schwerpunkten „Koordination und Qualität“, „Kooperation und Verantwortung“, „Koordination und Integration" bis zum jüngsten Gutachten „Wettbewerb an der Schnittstelle zwischen ambulanter und stationärer Gesundheitsversorgung “ wurde klar, dass Organisation und Strukturen des deutschen Gesundheitswesens sich nicht ausreichend an den gewandelten Erfordernissen einer Bevölkerung mit steigenden Prävalenzen chronischer Krankheiten in einer Gesellschaft des längeren Lebens ausgerichtet sind.

Die Organisation des Gesundheitswesens ist geprägt von heterogenen Verantwortlichkeiten und Strukturen, die letztlich einer notwendigen, an den Patienten ausgerichteten effektiven und effizienten Versorgungsstrukturierung entgegen stehen. Im Ergebnis stellt sich das heutige Organisationspuzzle als „organisierte Verantwortungslosigkeit“ (Gerlach) dar. Es ist wiederholt beschrieben, dass 
eine Verbesserung der Gesundheitsversorgung insgesamt auf die Lösung von Schnittstellenproblemen zwischen den Behandlungsarten-, -stufen und Leistungssektoren ausgerichtet sein muss. Dabei gilt es, im Rahmen von Kooperation und Integration jeweils die optimale Aufstellung (Produktionsprozess) unter Wirtschaftlichkeits- und Qualitätsaspekten bei Nutzung von Substitutionsmöglichkeiten zwischen den Bereichen zu finden. Diesem Anspruch sind nur dann Erfolgsaussichten zuzuschreiben, wenn nicht die Klein-klein-Lösungen verfolgt, sondern „organisierte Verantwortungslosigkeit" nochmals grundsätzlich angegangen wird. Ein punktuelles Reformieren der Regelungen im Bereich der so genannten besonderen Versorgungsformen $\left(\mathbb{S} \int 63-65,73 b, 73 c\right.$, 137f-g und 140a-d SGB V), etwa durch neue Förderprogramme, würde diesen Anspruch erkennbar verfehlen.

\section{Effizienzbetrachtung}

Zwar weist das deutsche Gesundheitssystem eine hohe Produktionseffizienz auf, d.h. bezogen auf den Mitteleinsatz werden auch viele Leistungen produziert. Auch die Kosten der einzelnen Leistungen sind im Vergleich zu anderen Industrienationen nicht überdurchschnittlich, d.h. es liegt im Allgemeinen auch eine hohe finanzielle Produktionseffizienz vor (Preisebene). Somit wäre zu erwarten, dass aus moderatem Preisniveau und sehr hohen Leistungsraten auch eine sehr hohe allokative Effizienz resultiert. Diese ist im OECD-Vergleich jedoch nur Mittelmaß, konkret: Der überdurchschnittliche Mitteleinsatz auch in Form sehr hoher Leistungsraten produziert keine überdurchschnittlichen gesundheitlichen Outcomes von zentraler Relevanz, wie Lebenserwartung, gesundheitlich unbeeinträchtigte Lebensjahre, Krebshäufigkeit etc. Vielmehr liegen die gesundheitlichen Outcomes im OECD-Mittelfeld.

Die Betrachtung der physischen Produktionseffizienz führt zu einer Erklärung. Die physische Produktionseffizienz bezeichnet das Behandlungsangebot im Sinne der Frage nach dem optimalen Einsatzverhältnis der verschiedenen Produktionsverfahren (z.B. Prävention, Beratung, Operationen, Rehabilitation, Hygiene, Pflege usw.). Dies impliziert Fragen danach, ob die richtigen Leistungen bzw. Verfahren an der richtigen Stel- le in der notwendigen Qualität und mit ausreichender Koordinierung eingesetzt werden. Es ist davon auszugehen, dass die so definierte physische Produktionseffizienz bzw. dieses Einsatzverhältnis in hohem Maße die letztlich für ein Gesundheitswesen maßgebliche Größe der gesundheitlichen Outcomes beeinflusst.

Dieser im Ergebnis für eine gute $\mathrm{Ge}$ sundheitsversorgung Ausschlag gebende Aspekt wird erst neuerdings durch die Etablierung von mehr Versorgungsforschung beleuchtet. Für die Umsetzung entsprechender Erkenntnisse und daraus resultierender Versorgungskonzepte wird die Notwendigkeit einer übergreifenden Verantwortlichkeit und Steuerungsfähigkeit theoretisch erkennbar und im Übrigen durch zahllose praktische Erfahrungen untermauert.

\section{3. Übergreifende Verantwortlichkeit}

Wer könnte diese notwendige übergreifende Verantwortlichkeit wahrnehmen? Unbestritten kommt den Bundesländern eine verfassungsgerichtlich untermauerte politische Verantwortung für die Sicherstellung einer bedarfsgerechten gesundheitlichen Versorgung der Bevölkerung zu, die anhand von Gesundheitszielen bzw. Indikatoren auf der Makroebene stets zu überwachen ist.

Die Option umfassender unmittelbarer staatlicher Steuerung wie in dezidiert staatlichen Gesundheitssystemen wäre zwar eine theoretisch begründbare Alternative, um die aufgezeigten Defizite anzugehen. Die Implikationen wären jedoch außerordentlich weitreichend, beginnend mit der Notwendigkeit neuer Abgrenzungen von Bundes- und jeweiligen Landeskompetenzen, quasi Abschaffung der Krankenkassen und Umwidmung in eine einheitliche Struktur nach dem Muster einer „Bundesagentur für Gesundheit“ inklusiv zahlreicher regionaler „Gesundheitsagenturen“, die die Versorgung vor Ort im Sinne von Managementgesellschaften zu organisieren hätten. Der Wettbewerb auf der Versicherungsseite wäre damit negiert, auf der Leistungsanbieterseite potentiell sogar erhöht, da einer vielfältigen Angebotsstruktur ein Nachfragemonopolist gegenüber stünde. Ob allerdings staatliche Institutionen die Kraft für versorgungsrationale Einzelentscheidungen im Gesundheitswesen aufbringen, kann bereits mit Blick auf
Entscheidungsprozesse zu Leistungsumfang oder Leistungsstandorten (z.B. in der Krankenhausplanung der Länder) mit Fug und Recht bezweifelt werden.

Die übergreifende staatliche Sicherstellungsverantwortung sollte sich deshalb im Wesentlichen auf eine Rechtsaufsichtspflicht über die unmittelbaren Akteure sowie Rahmenplanungsvorgaben beschränken. Die Konzentration und Beschränkung staatlicher Akteure auf die Etablierung sowie konsequente Überwachung eines geeigneten Ordnungsrahmens kann als Stärkung des Staates verstanden werden, der andernfalls Gefahr läuft, sich in mikroökonomischer Beteiligung am pluralistischen Interessenkampf aufzureiben und damit letztlich in ein Legitimitätsdefizit zu geraten. Als anschauliches Beispiel mögen die jüngsten Erfahrungen mit zahlreichen Landesbanken und der Erkenntnis des Vorrangs einer funktionierenden Bankenaufsicht gegenüber dem staatlichen Eigenbetrieb von Banken dienen.

Für die notwendige Steuerungsfunktion im Sinne einer effizienten Versorgungsstrukturierung insgesamt sind aufgrund sowohl ihrer gesetzlich wettbewerblichen Ausrichtung als auch ihrer inneren Organisationsstruktur einzig Krankenkassen geeignet. Sie könnten diese übergreifende Verantwortung wahrnehmen. Während etwa Krankenhausgesellschaften, Kassenärztliche Vereinigungen (KVen) oder Apothekerverbände natürlicherweise insbesondere individuelle und gruppenspezifische Interessen verfolgen und damit schon institutionell nicht auf eine Gemeinwohlorientierung programmiert sind, stellt sich dies für Krankenkassen anders dar: Der (sozialpartnerschaftliche) Selbstverwaltungscharakter der Krankenkassen spiegelt im Ergebnis sowohl die Zahler- als auch die Patientenperspektive wider und leistet bereits einen internen Interessenausgleich zwischen möglichst effizientem Mitteleinsatz und möglichst effektiver Versorgung. Unter den Bedingungen des freien Versichertenwettbewerbs i. V. m. dem morbiditätsorientierten Risikostrukturausgleich (mRSA) steht das Handeln von Krankenkassen im Sinne dieses Interessenausgleichs zudem unter unmittelbarer Sanktionsdrohung durch Kündigung und letztlich Insolvenz, ohne dass das solidarische System der GKV dadurch insgesamt gefährdet würde. 
Die konkrete Ausgestaltung der Versorgung sollte in der Konsequenz möglichst unmittelbar den Krankenkassen einerseits und den Leistungsanbietern anderseits in einem sozialstaatlich ausgerichteten grundsätzlich wettbewerblich geprägten Ordnungsrahmen übertragen werden. Bezogen auf die Diskussion zur Schaffung eines aus GKV und PKV integrierten Versicherungssystems würde diesem damit eine inhaltliche Fundierung gegeben, die sowohl das Gerede von der „Einheitskasse“ ad absurdum führen und über einen einst in Lahnstein propagierten reinen Service- und Vertriebswettbewerb weit hinaus reichen würde.

Krankenkassen sind die einzigen Akteure im Gesundheitswesen, die grundsätzlich zu allen Segmenten von Leistungsanbietern direkte Vertragsbeziehungen aufweisen, freilich mit sehr unterschiedlichen Regelungsintensitäten und Steuerungsmöglichkeiten. Damit sind Krankenkassen nicht nur hinsichtlich der Legitimität, sondern auch ganz praktisch die Einzigen, die prinzipiell die Voraussetzungen erfüllen (können), die verschiedenen Leistungssegmente im Sinne eines gestuften und rationalen Versorgungsprozesses aufeinander abzustimmen. Dafür ist ein Ordnungsrahmen zu schaffen, der die Krankenkassen konsequent in einen Wettbewerb um die beste Gesundheitsversorgung im skizzierten Sinne von aufeinander abgestimmten „Produktionsprozessen“ führt.

\section{Politische Handlungsanforderungen}

Nach politischen Ansätzen, die einzelnen Krankenkassen stärker in einer Rolle als Versorgungsgestalter zu etablieren, erfolgte spätestens mit dem Versorgungsstrukturgesetz eine (vorübergehende?) Restauration der zentralistisch korporatistischen Strukturen. Der Preis dafür bildet die weitere Verkrustung der Strukturen, die den eingangs skizzierten externen Anpassungsdruck immer ungenügender umsetzen können. Konfliktlösungsmechanismen fanden und finden demzufolge eher in Form zusätzlicher Finanzmittel denn in Strukturanpassungen statt, wie aktuell im Wahljahr mit der „Gießkannenförderung“ von Krankenhäusern erneut exemplarisch

unter Beweis gestellt wurde. Insofern bieten die üblichen hinlänglich bekannten kleinen Eingriffe in das Machtgefüge zwischen den korporatistischen Institutionen bzw. zwischen diesen und unmittelbar administrativen Eingriffen auch wenig Grund zur Annahme, damit letztlich den Anforderungen einer strukturierten Versorgung tatsächlich besser gerecht werden zu können.

Als verantwortlicher Hemmschuh einer schnelleren und intensiveren Umsetzung von sinnvoll strukturierten und integrierten Versorgungskonzepten erweist sich immer wieder eine generelle Systemträgheit. Ihre Ursachen scheint sie teilweise nicht zuletzt in einer finanziellen Saturiertheit der Beteiligten zu haben, die sich aus den eingangs skizzierten primär finanziell dominierten dysfunktionalen Handlungsstrategien in zentralistisch

Um den skizzierten Weg eines sinnhaften Versorgungswettbewerbs zwischen Krankenkassen einzuleiten, ist auch die ystemträgheit anzugehen.

gesteuerten verkrusteten Strukturen ableiten lässt. Um den skizzierten Weg eines sinnhaften Versorgungswettbewerbs zwischen Krankenkassen einzuleiten, ist auch die Systemträgheit anzugehen, woraus sich zunächst insbesondere nachfolgende Anforderungen an Politik und Gesetzgeber ergeben:

1) Die solidarisch aufgebrachten Finanzmittel (mRSA) können nur in einem solidarisch einheitlichen Leistungskatalog verwendet werden. Darüber hinausgehende Leistungen sind ggf. konsequent über individuell finanzierte Wahlbzw. Zusatztarife anzubieten. Hieraus ergeben sich auch Perspektiven einer Integration von PKV und GKV. Der solidarisch einheitliche Leistungskatalog wird abschließend vom Gemeinsamen Bundesausschuss (GBA) definiert. Damit erfolgt neben einer hervorragenden Service- bzw. Kundenorientierung eine Konzentration der Bemühungen auf optimale Versorgungslösungen anstelle der Ausweitung von neuen Leistungen, die sich häufiger an Wünschen von bestimmten Zielgruppen unter Deckungsbeitragsaspekten als an (evidenzbasierten) medizinischen Kriterien orientieren.
2) Die gesetzlichen Vorgaben zu einheitlich und gemeinsamen Vertragsabschlüssen von Krankenkassen mit Leistungsanbietern werden konsequent zurückgeführt, insbesondere im Bereich der vertragsärztlichen Versorgung. Die ambulant ärztlichen Teilleistungsbereiche Hausärztliche Versorgung, Fachärztliche Versorgung, Spezialärztliche Versorgung einschließlich des ambulanten Operierens, Psychotherapeutische Versorgung sowie Notfallversorgung (Notdienst) werden in eigenständige Verhandlungs- und Vergütungssäulen gefasst. Allein die Notfallversorgung ist krankenkassenübergreifend einheitlich zu verhandeln und zu gestalten.

3) Kontrahierungszwänge der Krankenkassen mit Leistungsanbietern werden neu geordnet, insbesondere durch Aufhebung für fachärztliche und spezialfachärztliche Leistungen sowie für bestimmte v.a. elektive stationäre Leistungen. Der Sicherstellungsauftrag wird in soweit grundsätzlich auf die Krankenkassen übertragen, die diesen durch entsprechende Vertragsabschlüsse erfüllen müssen. Die Gewährleistung dieser Verpflichtung wird über staatliche Aufsichts- und Eingriffsrechte auf Landesebene unterlegt.

4) Das Zulassungsrecht wird weiterentwickelt, insbesondere durch die Ermöglichung fachgebietsgleicher medizinischer Versorgungszentren (MVZ) z.B. rein hausärztlich - und Etablierung eines neuen Zulassungsstatus', der unter rein qualitativen Prüfkriterien auch eine ausschließliche Teilnahme an Selektivverträgen ermöglicht.

5) Die Bereinigungsregelungen im Bereich der vertragsärztlichen Versorgung sowie analoge Regelungen für den stationären Bereich werden weiterentwickelt und vereinfacht bzw. erstmals funktionsgerecht eingeführt. Wesentliche inhaltliche Eckpunkte der Bereinigungsregelungen insbesondere im Bereich der vertragsärztlichen Versorgung - soweit sie in einem neu gestalteten ambulanten Vergütungssystem auf der Basis einer GKV-PKV-Integration nicht ohnehin entbehrlich sind - sollten unmittelbar und abschließend vom Gesetzgeber erfolgen. Damit wird dem bestehenden Destruktionspotenzial der „Regelversorgungsakteure" die Grundlage entzogen.

6) Die Aufgaben der Selbstverwaltungspartner auf Bundesebene, insbesondere von DKG, KBV und GKV-SV werden konsequent auf eine Rahmen- 
gestaltung zurückgeführt, die insbesondere keine direkten Vergütungsvorgaben enthalten und strikt wettbewerbsneutral auf Ebene der Krankenkassen ausgerichtet sind. Demnach werden die Aufgaben auf Bundesebene bzw. im gemeinsameinheitlichen Zwangsmodus konsequent am Prinzip der Nachrangigkeit ausgerichtet. Nur solche Regelungen oder Aufgaben, die nicht unmittelbar zwischen einzelnen Krankenkassen und einzelnen Leistungserbringer(gruppen) gestaltet werden können, erfolgen auf einer entsprechenden zentralen Steuerungsebene.

7) Arzneimittel- und Apothekenrecht werden angepasst, um konkret auf rechtssicherer Basis erweiterte Abgabemöglichkeiten von Arzneimitteln durch Vertragsärzte und Krankenhäuser zu schaffen im Rahmen von entsprechenden Vereinbarungen mit Krankenkassen. Das (aufgeweichte) Mehrbesitzverbot bei Apotheken wird insgesamt aufgehoben, um die bestehenden, eher an Zunftordnungen des ausgehenden achtzehnten als an modernen Dienstleistungserfordernissen des einundzwanzigsten Jahrhunderts ausgerichteten, Angebotsstrukturen zugunsten von effizienten Betriebs- und Vertriebsformen abzulösen.

Die konkrete Ausgestaltung eines neuen gesetzlichen Ordnungsrahmens, der diesen Aspekten gerecht wird, bildet den versorgungseitigen Reformbedarf in der gesetzlichen Krankenversicherung ab.

\section{Referenzen}

Im Bereich der Gestaltung von Selektivverträgen und der zielgenauen Leistungssteuerung liegen bei der AOK BadenWürttemberg umfassende und evaluierte Erfahrungen zur Versorgungsgestaltung vor. Insbesondere die hausarztzentrierte Versorgung nach $\mathbb{}$ 73b SGB V in Verbindung mit Facharztverträgen nach \ 73c SGB V (derzeit in den Bereichen Kardiologie, Gastroenterologie, Psychotherapie, Psychiatrie, Neurologie und Orthopädie) bilden einen umfassenden strategischen und prozessualen Versorgungsansatz ab. Allen Verträgen ist gemeinsam, dass sie die für die jeweiligen Fachgruppen relevanten medizinischen Versorgungsbereiche anhand der qualitativ besten verfügbaren Leitlinien definieren und die Vergütungsstruktur sich vor allem an den Krankheitsbildern und nicht an einzelnen Verrichtungen ausrichtet. Damit werden ein verbindlicher Indikationsbezug des Handelns und somit gleichzeitig eine sorgfältige Diagnosestellung als Ausgangspunkt aller weiteren Therapieoptionen befördert. Wesentliches Merkmal der 73c-Vertragsgestaltung ist ferner, dass an diesen Verträgen nur Versicherte der AOK Baden-Württemberg teilnehmen können, die bereits in der HZV eingeschrieben sind. Denn HZV und 73c-Verträge sind strukturell miteinander verschränkt hinsichtlich inhaltlicher Therapiepfade und Schnittstellen der ärztlichen Tätigkeiten zwischen den Beteiligten.

Auch hinsichtlich der Kommunikation und Erläuterung der Vertragsinhalte und deren Umsetzung beschreitet die AOK Baden-Württemberg mit dem kassenseitigen Arztpartner-Service (APS) neue Wege. Die dezentral in ganz BadenWürttemberg aufgestellten Mitarbeiterinnen und Mitarbeiter stehen den teilnehmenden Praxen als Berater, Kümmerer und „Vernetzer“ zur Verfügung. Sie leisten über persönliche Kommunikation vor Ort ihren Beitrag zu einem partnerschaftlichen Miteinander und einer erfolgreichen Vertragsumsetzung. Weitere Anknüpfungspunkte einer strukturierten Versorgung sind unmittelbare Angebote der AOK wie z.B. deren Gesundheitsangebote oder Beratungsangebote des Sozialen Dienstes zur Herstellung von stabilen Gesamtversorgungssituationen (ausführlicher auf www.hzv-aktuell.de sowie Hermann/Graf in Gesundheitsund Sozialpolitik 6/2012).

Die Weiterführung der spezifisch an Qualitätskriterien und gemeinsamem Kooperationsverständnis ausgerichteten Versorgungspfade in angrenzende Leistungsfelder liegt auf der Hand, bedarf jedoch der aktiven Gestaltung auf der Grundlage - derzeit fehlender - praxistauglicher rechtlicher Kompetenzen.

\section{Gesetzgeberische Sofortmaßnahmen}

Die bislang erzielten Erfolge selektivvertraglicher Versorgungsgestaltung beruhen auf einer konstruktiv vertrauensvollen Zusammenarbeit der Vertragspartner und erfordern so viel Geduld wie Ausdauer als auch - wettbewerblich motivierte - Innovationsfreude. Von hoher Bedeutung ist dabei die unmittelbar gelebte Verantwortungsübernahme für die regionale Versorgung durch die AOK
Baden-Württemberg im Wege der Übernahme des Sicherstellungsauftrages im Rahmen von HZV und 73c-Verträgen gemeinsam mit den Partnern Hausärzteverband und MEDI sowie verschiedenen unterstützenden Berufsverbänden.

Auch vor diesem Hintergrund stellt der durch das GKV-FinG 2011 eingeführte Investitionsvorbehalt in $\mathbb{S} 73 \mathrm{~b}$ Abs. 5a SGB V eine Gefährdung für die Weiterentwicklung nachweislich erfolgreicher hausarztzentrierter und darüber hinausweisender Versorgungsstrukturen dar. Er sollte unmittelbar ersatzlos gestrichen werden, da er Innovation über Investitionen verhindert. Zielführend wäre es im Weiteren, die skizzierten sieben Reformpunkte gesetzgeberisch aufzugreifen, um das Potential wettbewerblicher Suchprozesse für optimierte Versorgungsprozesse zu erschließen.

Einen pragmatischen und rasch realisierbaren Einstieg für einen fließenden Übergang in eine breitere Verantwortungsübernahme durch Krankenkassen stellt ein 2-Säulen-Konzept dar, das in einem Optionsmodell für definierte Versicherte breitere Gestaltungsmöglichkeiten eröffnet. Das entsprechende Versichertenkollektiv könnte konkret der Versichertengruppe entsprechen, die sich freiwillig auf der Grundlage der Teilnahme an der hausarztzentrierten Versorgung in ein darüber hinaus reichendes Versorgungskonzept ihrer Krankenkasse begibt. Breitere Gestaltungsspielräume wären etwa veränderte Zulassungsoptionen zur Mitwirkung für Ärzte sowie erweiterte Abgabe- und Distributionsmöglichkeiten für Arzneimittel. Daneben sollte klargestellt werden, dass der Bereinigungsumfang (vgl. \ 73 b Abs. 7 Satz 1 SGB V) den konkret selektivvertraglich erbrachten Versorgungsumfang überschreiten kann. Dadurch können Effizienzsteigerungen durch die selektivvertragliche Versorgungssteuerung auch unmittelbar dieser finanziell zugeordnet werden.

Aufgrund der Freiwilligkeit und des vorauszusetzenden Konsenses zur Gestaltung des Gesamtversorgungskonzepts insbesondere mit den hausärztlichen Partnern wäre sichergestellt, dass Koordination, Kooperation und Qualität im Sinne der eingangs skizzierten physischen Produktionseffizienz zu den Fixpunkten einer entsprechenden Versorgungsgestaltung würden.

Literatur bei den Verfassern 